\title{
Decreased $b$-value prior to the $M$ 6.2 Northern Miyagi, Japan, earthquake of 26 July 2003
}

\author{
Yoshiki Tsukakoshi* and Kunihiko Shimazaki \\ Earthquake Research Institute, University of Tokyo, 1-1-1 Yayoi, Bunkyo-ku, Tokyo 113-0032, Japan \\ (Received September 25, 2006; Revised May 26, 2008; Accepted June 4, 2008; Online published October 15, 2008)
}

\begin{abstract}
We developed a method to map and investigate temporal changes in the $b$-value using the signed Akaike information criterion (AIC) value and seismicity rate before the 2003 September 26-27 Northern Miyagi earthquake sequence. We studied the seismicity within a radius of $30 \mathrm{~km}$ around the epicenter of the largest shock, which was $M 6.4$, by three approaches: an analysis of all the events listed in the catalog; an analysis of only background events, but on data extracted by two different methods. We found that the change in the $b$-value of the clustered activities and background activities are different. The $b$-value of all events decreased from 1.2 to 0.7 and that of background event decreased even more. We empirically modeled the precursory temporal variation of the $b$-value and searched similar patterns among 971 areas chosen sequentially from inland Japan. The precursory pattern was both rare and significant; the AIC value was the second most significant factor among the more than 3000 tests conducted on 971 areas, each with four time windows. If the catalog was not declustered, the AIC of the precursory pattern became lower.
\end{abstract}

Key words: $b$-value, precursory, ETAS model, seismicity.

\section{Introduction}

Although some doubt their significance of precursory seismic patterns before large earthquake, there have been a number of reports on such patterns, including precursory swarm or preshocks (Sekiya, 1976), accelerating seismic moment release (Varnes, 1989; Sykes and Jaume, 1990), precursory seismic quiescence (Inouye, 1965), decreases in $b$-value (Imoto, 1991), donut pattern (Mogi, 1969), horizontal migration (Kagan and Knopoff, 1976), downward migration (Mogi, 1988), and bursts of aftershocks (KelisBorok et al., 1980). Rundle et al. (2002) obtained some positive results on the possibility of earthquake prediction based on seismicity pattern. Such patterns should be investigated given the impact of earthquake prediction to society. As seismic activity reflects the physical condition of Earth's crust, any information relating to forthcoming, potentially damaging earthquakes is of importance.

Temporal variations in $b$-values and their relation to the forthcoming damaging earthquakes have been discussed by many authors. Cases where the $b$-value decreases before large earthquakes have often been reported. Wyss and Lee (1973) reported a decrease in the $b$-value before four out of five California earthquakes with magnitudes of 3.9 to 5.0. Smith $(1981,1999)$ found that an increase in the $b$ value was present for several years before the occurrence of large earthquakes and that the $b$-value decreased to normal

\footnotetext{
*Current position: National Food Research Institute, National Agriculture and Food Research Organization, Tsukuba, Japan.

Copyright (c) The Society of Geomagnetism and Earth, Planetary and Space Sciences (SGEPSS); The Seismological Society of Japan; The Volcanological Society of Japan; The Geodetic Society of Japan; The Japanese Society for Planetary Sciences; TERRAPUB
}

in the short interval before the large event itself. Ogata and Katsura (1988) also found low $b$-values before large aftershocks. Li et al. (1978) found a decrease in the $b$-value before the Tanshang earthquake, and Imoto (1991) reported a decrease in the $b$-value before several earthquakes that occurred in inland Japan. Hirose et al. (2002) studied the temporal variation of $b$-values in asperities of subduction zones and found that a significant decrease in the $b$-value occurred in an asperity (asperity $b$ in their paper). Imoto (2003) developed a testable model based on an increase in mean event size (equivalent to a decrease of the $b$-value) and obtained a probability gain of two- to fourfold in the Kanto area, Japan.

A decrease in the $b$-value prior to large earthquakes has also been observed in numerical simulations (Yamashita and Knopoff, 1992; Hainzl et al., 2003) and laboratory experiments (Scholz, 1968; Ohnaka and Mogi, 1982; Sammonds et al., 1992; Lei et al., 2004), which realized several well-founded properties of seismicity, such as Gutenberg-Richter's magnitude-frequency relation (Gutenberg and Richer, 1944) and modified Omori's law (Utsu, 1961). See Main et al. (1989) and Main (1996) for a comprehensive review on these subjects.

Several earthquake prediction trials based on these precursory patterns have been proposed and tested. The application of a $b$-value model (Imoto, 2003) and M8 algorithm (Kellis-Borok and Kossobokov, 1990) shows that these patterns can improve the time-dependent earthquake hazard models. However, these algorithms also produce an ineligible number of false alarms, clearly illustrating that more knowledge on seismic activity is required.

An increase in the number of detailed case studies on precursory patterns is expected to result in empirical in- 
formation that will improve the time-dependent earthquake hazard model. Although very wide variations in the maturity, inhomogeneity, geometry, and physical condition of the seismogenic faults exist, the number of case-studies is too small to enable any expert discussion of the relation of seismicity patterns, including precursoric ones, to such differences.

We studied the seismicity prior to the Northern Miyagi sequence. The $b$-value change was investigated by separating the total event into background events and their aftershocks with the aim of identifying new patterns. We consider this separation to be important because the $b$-value of the aftershocks can behave differently from those of the background events. Considering the $b$-value of aftershocks in a sequence is often temporally invariant (e.g., Utsu, 1961), as the $b$-value of aftershock may depend on the mainshock and not on the forthcoming earthquakes.

\section{Data}

Our data were drawn from the JMA (Japan Meteorological Agency) catalog. From October 1997 up to June 2003, the completeness level of magnitude is considered to be smaller than 2.0 for the most inland part of Japan (Ohta $e t$ al., 2004).

Several changes in the observatory networks occurred during our study period. In October 1997, the JMA began to collect seismograms that had been installed by various universities and institutes throughout Japan. In October 2001, the High Sensitivity Seismograph network commenced operations in the territory of the Sendai district meteorological observatory, which covers the study area. We paid attention to these changes and show the results with $b$-value changes by natural causes in a subsequent section of this article. We ultimately concluded that these changes did not affect the reporting of earthquakes with a magnitude $\geq 2.0$.

Using a consistent magnitude scale is essential when discussing $b$-value changes because the use of different magnitude scales would give different $b$-values. Here we mostly used the old JMA magnitude scale and applied the newer one (Katsumata, 2004) as a supplement despite the recent revision of the JMA magnitude scale. We did this because the revised magnitude may be temporally inhomoge- neous. As the magnitude scale is sensitive to the observatory network (e.g., Habermann and Craig, 1988; Mashiko and Noguchi, 2003), the new stations installed during our study period (from 1997 to 2003) may induce systematic change in the reporting of the magnitude scale. Also, small earthquakes are attached greater weights when the $b$-value is estimated by the maximum likelihood estimate compared to least square estimation (Utsu, 1965). To avoid misunderstanding; we should be careful that the relative insensitivity of the estimated parameter $b$ to the number of large events does not mean that the number of large events does not affect the problem of model selection at all.

We selected events shallower than $20 \mathrm{~km}$ in order to remove deep events from the data. The depth was selected because the subducting plate boundary is deeper than $20 \mathrm{~km}$ in most of inland Japan. We used events with a precision flag ' $\mathrm{K}$ ', which means the epicenter is reliable. While this precaution prevented our counting wrong events, most events in the catalog with a magnitude $>2.0$ are marked with the ' $\mathrm{K}$ ' flag, and our step may therefore be considered almost redundant.

\section{Method and Model}

\subsection{Gutenberg-Richter's relation and cumulative mag- nitude plot}

The representation of Gutenberg-Richter's law (Gutenberg and Richter, 1944) for frequency-magnitude distribution can be expressed by the following probability density function.

$$
P(M \mid b)=\left(\log _{10}(e) / b\right) 10^{-b M}
$$

where $M$ is the magnitude of the event, $b$ is a constant, and $P(M \mid b)$ is the probability density function.

The maximum likelihood estimate of the parameter $b$ is given by the following simple equation

$$
\tilde{b}_{\text {m.l.e. }}=\log _{10}(e) /\left(E[M]-M_{0}\right)
$$

(Aki, 1965), where $E(M)$ denotes the average of magnitude of the sample, and $M_{0}$ denotes the lower magnitude threshold.
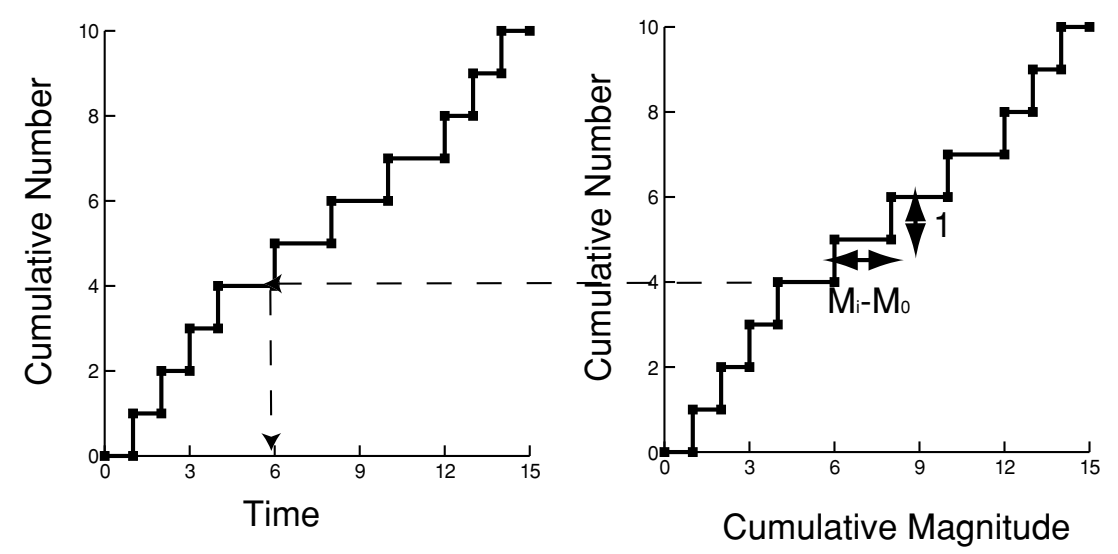

Fig. 1. Explanatory figure of cumulative magnitude plot. Using this plot, the slope of the plot coincides with the maximum likelihood estimate of the $b$-value. Also, the time of the $b$-value change can be read by using the cumulative number plot drawn with the same vertical axis. 
Smith (1986) studied $b$-value change by a method called CUSUM. The $y$-coordinate of CUSUM is given by

$$
C_{n}=\sum_{i=0}^{n-1}\left(M_{i}-\bar{M}\right),
$$

where $\bar{M}$ is the average of the magnitude of the entire sample or an approximation of the average. Instead, we subtract the magnitude of the lower threshold to find the changes in the $b$-value. This makes the gradient of the plot the reciprocal of the $b$-value (Faculty of Science, Tohoku University, 1991). If we swap the $x$ - and $y$-axis, we obtain the $b$-value itself. In other words, we plot on the point $\left(x_{i}, y_{i}\right)=\left(\sum_{j=0}^{i}\left(M_{j}-M_{0}\right), i\right)$ (Fig. 1). The gradient of the plot is then given by:

$$
\begin{aligned}
\delta y / \delta x & =i /\left(\sum_{j=0}^{i}\left(M_{j}-M_{0}\right)\right) \\
& =1 / E\left[\left(M-M_{0}\right)\right] \\
& =\log _{e}(10) \hat{b}_{\text {m.l.e. }}, \quad e=2.7182 \cdots .
\end{aligned}
$$

The advantage of this method is that we can understand the time of the $b$-value change easily by comparing the plot to the cumulative number versus the time plot. We can choose the same vertical axis of the above plot and the cumulative number plot because both vertical axes are the cumulative number of events (Tsukakoshi et al., 2007, 2008).

\subsection{Treatment of aftershocks}

3.2.1 Clustered activities and $b$-value Intensity functions are expressed by the sum of two terms: independent and their aftershocks.

We removed aftershocks because the lower level of magnitude completeness becomes significantly higher after large events occur (e.g., Kagan, 2004). Due to the longlasting coda wave, the arrival phases of small earthquakes can not be picked, and their hypocenters are therefore not

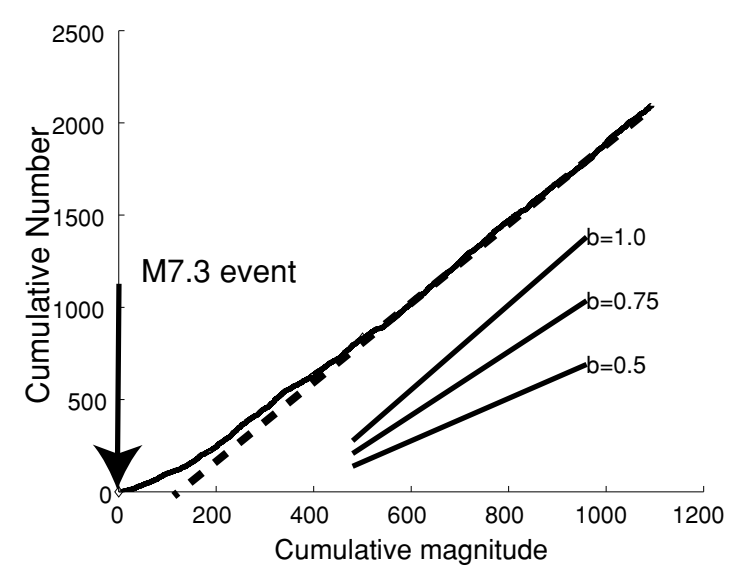

Fig. 2. Apparent $b$-value changes after the Western Tottori earthquake. The downward arrow shows the occurrence of the $M 7.3$ event. The $b$-value is low after the event, but this is due to the detectability of small events. The slope recovers at about the thousandth event, which means that small events are picked. After the thousandth event, the slope is constant until the end of the graph, which indicates that the $b$-value is almost constant during the sequence. determined. The apparent $b$-value becomes smaller but does not reflect actual seismic activity. For example, Fig. 2 shows the cumulative magnitude plot that includes the 2000 $M 7.3$ Western Tottori earthquake and its aftershocks. The tip of the downward arrow in the figure shows the occurrence time of the mainshock. The cumulative magnitude curve bends at the occurrence time and the slope becomes gentle. This means that the calculated $b$-value was temporally low after the event. The low $b$-value estimated by the gentle slope is not generally a natural phenomenon but, rather, caused by the indefectibility of the aftershocks. The absence of a small event increases the mean magnitude and decreases the estimated $b$-value.

Nevertheless, some authors have made several observations on using the declustered catalog to calculate $b$-value (Knopoff et al., 1982; Frohlich and Davis, 1993; Lombardi, 2003). They claim that many declustering procedures intentionally retain large events and discard smaller ones. This selection biases the estimated $b$-value, but it is not a serious problem in our study because we apply the same method to the whole period. The bias of two periods would therefore be equal.

3.2.2 Declustering method Two declustering methods have been adopted. Because the background events can be defined in many ways, the result would be affected to a greater of lesser extent by the definition that is used. Consequently, our approach of using two methods is very meaningful. One of the algorithms we adopted is Reasenberg's algorithm (Reasenberg, 1985), and the other is based on the space-time extended ETAS model (Ogata, 1998). These two algorithms have completely different methodologies. The Reasenberg's algorithm determines cluster based on the time and distance between events, while the ETAS model determines background seismicity based on the stochastic model of background and clustered activity.

For the Reasenberg's algorithm, we used the following options: Rfact $=10$ and an adjustment of the hypocentral errors. The source code was obtained from USGS's homepage (URL: http://quake.wr.usgs.gov/research/software/). We made minor modifications on it to deal with the format of the JMA catalog and the Y2K problem. There are about 200,000 events with a magnitude of not less than 2.0 in the catalog and about 120,000 clusters are reported in the period from October 1997 to June 2003.

3.2.3 Estimating the $b$-value using ETAS model The methodology of using the space-time ETAS model to extract background event information is the same as that described by Tsukakoshi and Shimazaki (2006) except that we considered magnitude-frequency distribution in our study. A brief outline of the methodology is as follows. If we denote the probability density function (p.d.f.) on the spacetime distribution of earthquake occurrence by $P(x, y, t)$, the total distribution function is given by a simple multiplication theorem for probability:

$$
P(x, y, t, M)=P(x, y ; M) P(M) .
$$

Let the probability density function of earthquake occurrence defined on the study area and period be $\mu+K$, where $\mu$ is the p.d.f. of the background event and $K$ is the p.d.f. of the aftershocks. Assuming that the two types of earth- 
quake has different $b$-values, we obtain the following p.d.f. for total seismicity.

$$
\begin{aligned}
\lambda(x, y, t, M)= & \beta_{\text {background }}(t) \exp \left(-\beta_{\text {background }}(t) M\right) \mu \\
& +\beta_{\text {aftershocks }}(t) \exp \left(-\beta_{\text {aftershocks }}(t) M\right) \\
& \times K(x, y, t) \quad\left(\beta_{\bullet}=b_{\bullet} \log _{e} 10\right)
\end{aligned}
$$

Using Eq. (1), we estimated the parameter $b_{\text {background }}$ and $b_{\text {aftershocks }}$, by maximizing the likelihood function for the above intensity function. The $b$-values are not fixed and estimated for each case.

There is one important difference between the significance of the $b$-values of the background events estimated by using the two declustering methods. Reasenberg's algorithm selects the largest event in the cluster as the mainshock, but the ETAS model holds immigrants or the first one in the cluster. There is also a difference in the determination method of the magnitude of the mainshock. The magnitude of main events calculated from the former is obtained by summing up the moment of each and every earthquake in the cluster; the latter simply takes the magnitude of first event. If these two methods give similar results, the result is reliable.

To draw a cumulative magnitude plot of the background event using ETAS model, we need additional information because the model does not determine the background event explicitly. Instead, we can use the fact that the model gives the probability of independence for event $i$ as below:

$$
\begin{array}{r}
p_{i}=\frac{\left(\beta_{\mathrm{b}}\left(t_{i}\right) \exp \left(-\beta_{\mathrm{b}}\left(t_{i}\right) M_{i}\right) \mu\right)}{\left(\beta_{\mathrm{b}}\left(t_{i}\right) \exp \left(-\beta_{\mathrm{b}}\left(t_{i}\right) M_{i}\right) \mu\right)+\left(\beta_{\mathrm{a}}\left(t_{i}\right) \exp \left(-\beta_{\mathrm{a}}\left(t_{i}\right) M_{i}\right) K\left(x_{i}, y_{i}, t_{i}\right)\right)}, \\
\beta_{\mathrm{b}}=b_{\text {background }}, \beta_{\mathrm{a}}=b_{\text {aftershocks }},\left(\beta_{\bullet}=b_{\bullet} \log _{e} 10\right),
\end{array}
$$

(Dempster et al., 1977; Kagan, 1999; Zhuang et al., 2004). Using this probability, we defined the $x-y$ coordinates of the point of cumulative magnitude as $(x, y)=$ $(\operatorname{sum}(p), \operatorname{sum}(p M))$. The slope of the plot gives the reciprocal of the weighted mean of magnitude, and the slope of the curve obtained using the entire sample coincides with $b_{\text {background }}$.

The mathematical justification of the procedure is given as follows. The log-likelihood of a point-process model is given by

$$
\log L=\log \lambda_{i}-\int_{R} \lambda d r
$$

where $R$ is the support of the intensity function $\lambda$ (a support is a subspace of the domain of a function where $\lambda$ has non-zero value; e.g., Ogata, 1998). Where the loglikelihood takes the maximum value, the optimal parameters of $b_{\text {background }}$ are given. Then, differentiating both sides of the equation, the left side of the equation becomes 0. Substituting Eq. (1) into Eq. (2) and differentiating by $b_{\text {background }}$ gives

$$
b_{\text {background }}=\log _{10}(e) \sum_{i} p_{i} /\left(\sum_{i} p_{i}\left(M_{i}-M_{0}\right)\right) .
$$

Thus, the slope of plot $(x, y)=\left(\operatorname{sum}\left(p_{i}\right), \operatorname{sum}\left(p_{i} M\right)\right)$ becomes the $b_{\text {background }}$ if $p$ is calculated using the maximum likelihood estimate of $b_{\text {background }}$. It may initially appear confusing to speak about the change in the $b$-value using the probability obtained by assuming a constant $b$ value is constant, however, we expect the slope is close to

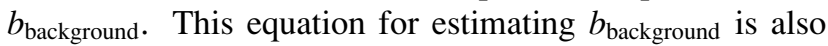
given in Zhuang et al. (2004) based on the consideration of probability. In this study, the relation between the equations to the maximum likelihood estimate was revealed, and we applied it to the cumulative magnitude plot to study temporal variations of $b_{\text {background }}$.

\section{Precursory Patterns of the Northern Miyagi Event}

\subsection{The 2003 September 26 Northern Miyagi event}

The Northern Miyagi earthquake sequence can be described as follows. The mainshock took place at 7:16 26 July 2003. According to the National Research Institute for Earth Science and Disaster Prevention, it was an intraplate shallow crustal event with a depth of $12 \mathrm{~km}$. It had a reverse-type mechanism, and the magnitude of the mainshock is estimated to be 6.4 in the JMA catalog. An M 5.5 event preceded the main event by $7 \mathrm{~h}$. The foreshock occurred in close vicinity of the epicenter of the mainshock, to the north, but its rupture zone spread south, close to the source region of the mainshock (Yagi et al., 2003; Hikima and Koketsu, 2004). The moment tensor mechanism of both the foreshock and the mainshock was of the reverse type. We can consider that the foreshock triggered the mainshock because the distance from the rupture area of the foreshock is close to the epicenter of the mainshock. The southern part ruptured first because it is heterogeneous and weak.

The foreshock-mainshock-aftershock sequence has a rare property in that the magnitude of the foreshock is large and the difference between the magnitudes of the foreshock and mainshock are very small-only 0.8. Considering the 1995 M 7.3 Southern Hyogo earthquake had foreshock whose magnitude was only 3.3, this difference of Miyagi event is very small. According to JMA (2003), between 1996 and 2003, there have been 163 sequences where the first event has a magnitude greater than 5.5 and shallower than $30 \mathrm{~km}$. However, there are only three cases where the following event is larger than the first and the event is separated by more than $20 \mathrm{~km}$ from the quaternary volcano.

\subsection{Time evolution of the $b$-value and seismicity rate- inclusion and removal of aftershocks, respectively}

A cumulative magnitude plot of the source region prior to the Northern Miyagi event is shown in Fig. 3. As seen from the slope of the plot (keeping in mind that the slope is the $b$-value), the $b$-value suddenly decreases at the end of 2002 and remains low during 2003. The $M 6$ events occurred on 26 June 2003. It may appear that the $b$-value decrease started early in 2002, but this is not correct. The right plot shows the time versus cumulative number plot where the vertical axis is identical to the left axis. From this, it is apparent that most of the seismicity observed in 2002 is from swarm activity at the end of 2002. The lower two plots show the cumulative magnitude plot of background events only. We studied background seismicity by the two methodologies described above. While the number of earthquakes decreases with removal of the clusters, the change in the slope is very distinct. We do not use data before 1998 because the change point in the $b$-value coincides with the time when 
a)No declustering

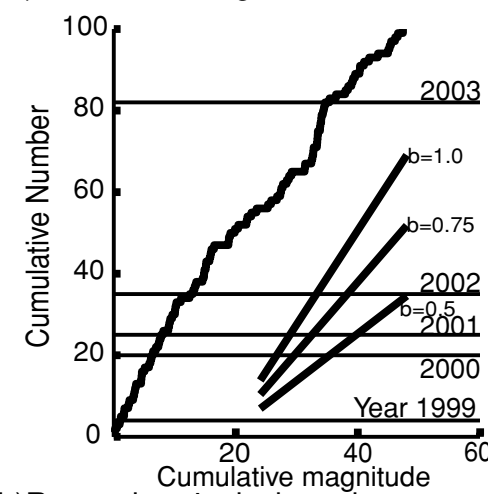

b) Reasenberg's declustering

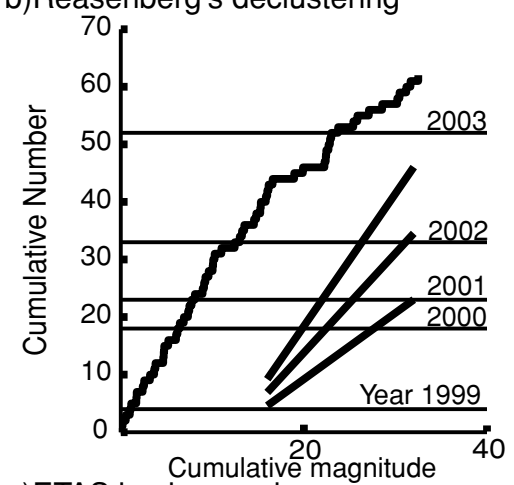

c)ETAS background

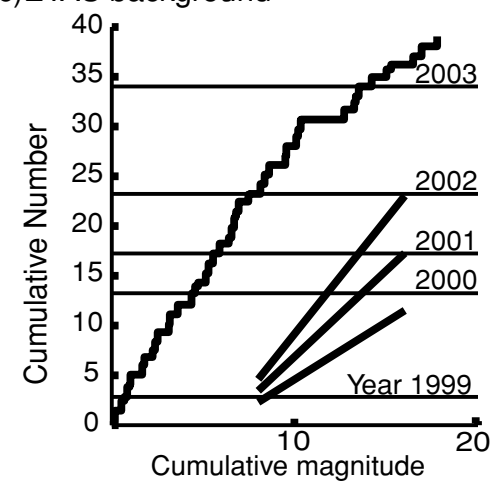

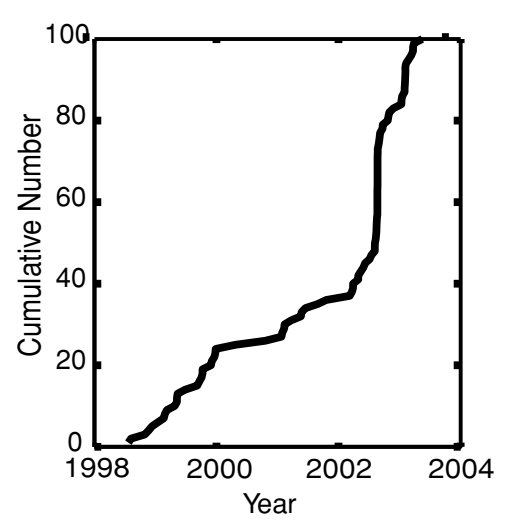
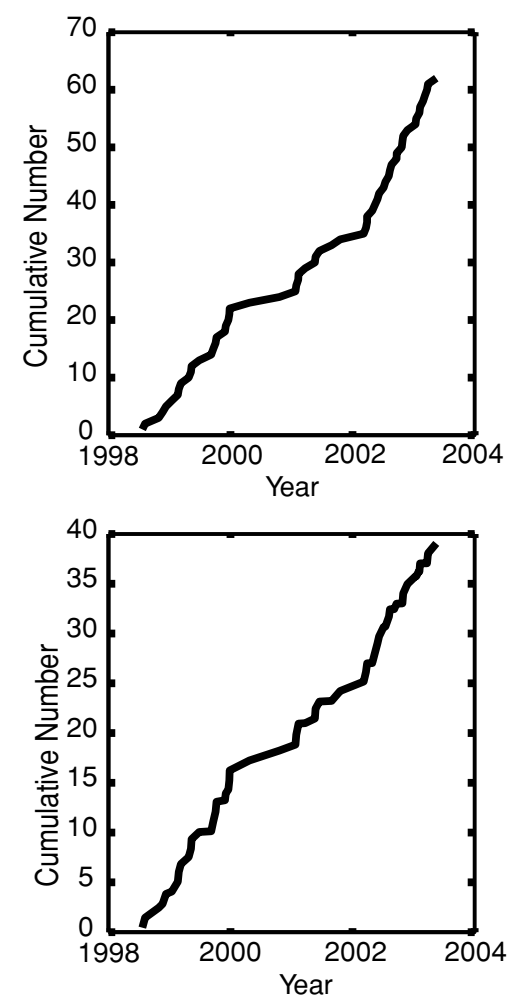

Fig. 3. Cumulative number plot versus cumulative magnitude plot before the Northern Miyagi earthquake. (a): cumulative magnitude of all events. (b): cumulative magnitude of declustered event from Reasenberg's algorithm. (c): the background event from ETAS model.

new observatory stations were installed.

\subsection{Modeling the precursory anomaly}

We first modeled this pattern in a very simple way: 4 years of a high $b$-value period followed by 1 year of a low $b$-value period. This model is described as:

$$
\begin{aligned}
B_{\text {background }}(t)= & B_{1} \operatorname{boxcar}(t ; 1998.5,2002.5) \\
& +B_{2} \operatorname{boxcar}(t ; 2002.5,2003.5)
\end{aligned}
$$

where boxcar $(t ; 1998.5,2005)$ denotes a boxcar function that is applied from July 1998 to June 2002; otherwise zero is used. The calculated $b$-value of the former period $\left(b_{1}\right)$ is 1.2 ; we obtained 0.7 for the latter $\left(b_{2}\right)$ without discriminating background events and aftershocks.

In the circular area with radius $30 \mathrm{~km}$ centered by $141.4 \mathrm{E}$, $38.4 \mathrm{~N}$, the $\log N-M$ plot is straight and the G-R relation holds very well (Fig. 4) for a magnitude $>2.0$. (We can not discuss magnitude below this range because the completeness of reporting was not adequate.) Some authors discuss the deviations from Gutenberg-Richter's law prior to large fractures on the source fault (Rundle et al., 2000). Such an effect was not apparent in our seismicity patterns near or on the extension of the source fault nor on the source fault itself.

The null hypothesis for the existence of change can be described as follows;

$$
B_{\text {background }}(t)=B_{0} \operatorname{boxcar}(t ; 1998, T) .
$$

The difference in the AIC (Akaike, 1974; Utsu, 1992) is 6.7 for the $b$-value change testing of the background events extracted by the Reasenberg's decluster method. The AIC using the ETAS model was 7.3. When we test the $b$ value change of both background and aftershocks (without declustering), the AIC difference is 3.9. Although the decrease in the $b$-value is significant in this case, the removal of the aftershocks from the data increases the AIC value.

The change in the $b$-value seems to have a very fast onset 


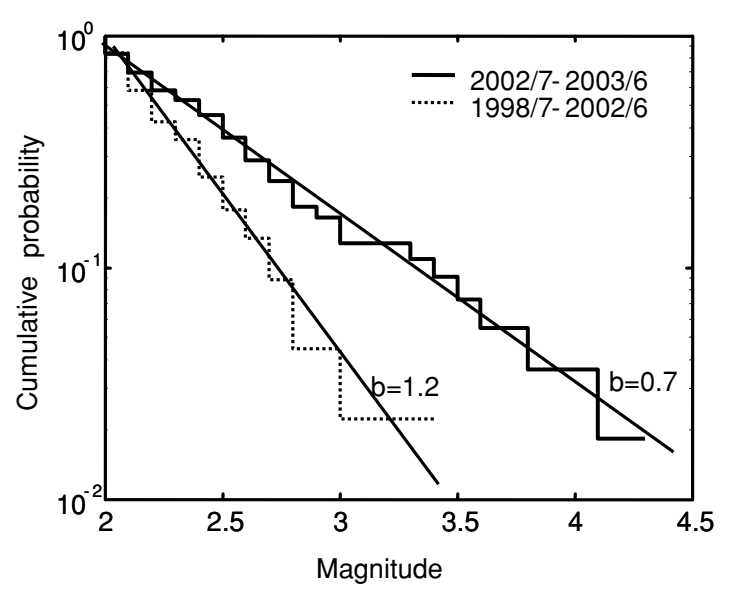

Fig. 4. Comparison of the magnitude-frequency relation of two periods: July 2002 to June 2003, and July 1998 to June 2002. The $b$-value of the former period is lower than that of the latter. In the magnitude range from 2.0 to 3.0, the line of the plot is linear, and the Gutenberg-Richter's law holds well.

time; as such, the boxcar function is more suitable than smooth functions, such as spline functions. Similar fast onset times of the precursory event have often been reported (Ohtake, 1980; Wyss and Habermann, 1988).

The seismicity rate was also changed and was bumped up due to swam activity. Accelerating seismicity on adjacent faults, which was pointed out previously by Varnes (1989), was found in this region.

The background seismicity rate has been modeled such that it has a quiescence period of 2 years.

$$
\begin{aligned}
1 \mu_{\text {background }}(t)= & \mu_{1} \operatorname{boxcar}(t ; T-5, T-3) \\
& +\mu_{2} \operatorname{boxcar}(t ; T-3, T-1) \\
& +\mu_{1} \operatorname{boxcar}(t ; T-1, T) \\
2 \mu_{\text {background }}(t)= & \mu_{0} \operatorname{boxcar}(t ; 1998, T)
\end{aligned}
$$

The first model assumes a quiescent period of 2 years and the recovery of the background level within 1 year. Model (1) is better than the constant background level model (Model 2) by an AIC difference of 3. Although this difference is statistically significant, it would not be discriminated beforehand from other random fluctuations of seismicity rate that are not followed by large earthquakes (Tsukakoshi and Shimazaki, 2006).

\subsection{Validation of the model}

The hypothesis that there was a decrease in the $b$-value prior to the Northern Miyagi event is also tested by applying different magnitude thresholds (Table 1), as is the revised magnitude scale. The decrease in the $b$-value is significant for a magnitude threshold higher than 1.8 in the catalog used in this study and 1.0 for the revised magnitude; these represent the completeness level of the magnitude. The differences are due to new magnitudes tending to be smaller than the earlier ones. The Data Analysis Section, Earthquake Prediction Information Division, Seismological and Volcanological Department, JMA (2004) reported a difference of 0.6 for events with a magnitude of 2.0. A higher magnitude threshold restricts the number of events that can be used for the analysis and would provide a decrease in the $b$-value that can be tested at only a low significance level.

As a case study of the decrease in $b$-values before a large earthquake, the result from the other spatial window used for sampling is shown in Table 2 . When we apply a $20-\mathrm{km}$ radius for sampling, a decrease in the $b$-value is found. The AIC is smaller than 2.0, but the sample size is too small to enable any discussion of the significance.

The decrease in the $b$-value can not be accounted for by the improvement in the observatory system. This conclusion can be drawn not only because we carefully selected lower magnitude thresholds and there was no sign of any incomplete reporting of events in the cumulative-number versus magnitude plot (Fig. 4) but also because the improvement will increase the number of small events, thus increas-

Table 1. The SAIC (a parameter which shows the significance of the $b$-value decrease) and the lower magnitude threshold applied for analysis.

A) Old magnitude scale

\begin{tabular}{ccc}
\hline Magnitude range & Background + event aftershocks & Background events \\
\hline$M \geq 2.2$ & -3.0 & -5.9 \\
$M \geq 2.0$ & -4.0 & -7.4 \\
$M \geq 1.8$ & -5.7 & -4.5 \\
\hline
\end{tabular}

B) Revised magnitude scale

\begin{tabular}{ccc}
\hline Magnitude range & Background event + aftershocks & Background events \\
\hline$M \geq 1.6$ & -1.8 & -5.1 \\
$M \geq 1.4$ & -6.0 & -4.6 \\
$M \geq 1.2$ & -4.9 & -5.2 \\
$M \geq 1.0$ & -3.8 & -3.5 \\
\hline
\end{tabular}

Table 2. Relation between sampling radius and the SAIC.

\begin{tabular}{ccc}
\hline Sampling radius (Number of events) & Background + event aftershocks & Background event \\
\hline $20 \mathrm{~km}(N=22)$ & -0.7 & -1.2 \\
$30 \mathrm{~km}(N=68)$ & -4.0 & -7.4 \\
$40 \mathrm{~km}(N=134)$ & -4.5 & -1.5 \\
\hline
\end{tabular}


a)

b)

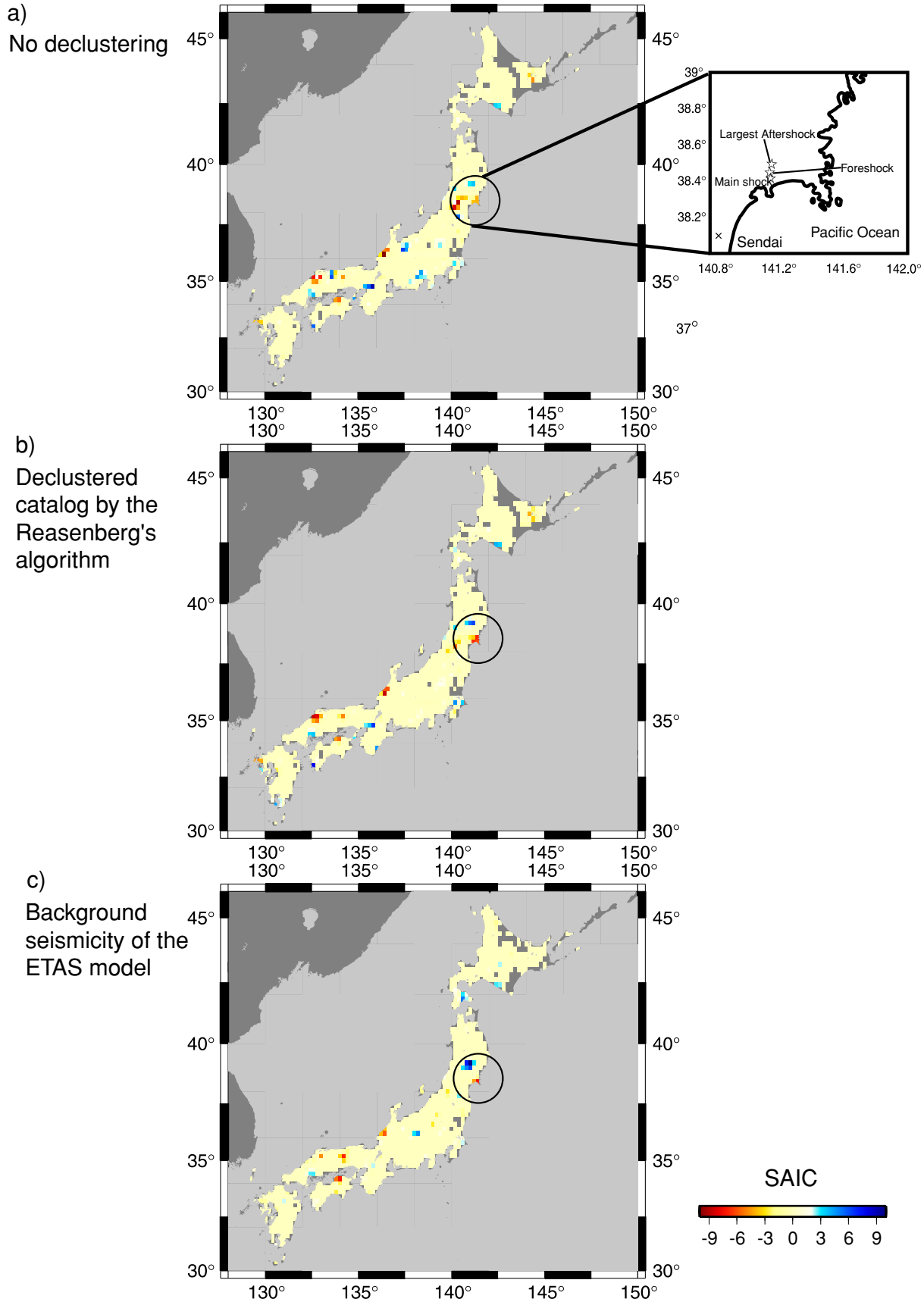

Fig. 5. Map of $b$-value change. The $b$-value change is estimated by SAIC defined in the text. The three figures each show a different declustering scheme. In most cases, the lower figure is the result obtained by removing triggered events using the ETAS model (Ogata, 1998) and the middle figure is the process by Reasenberg's algorithm (Reasenberg, 1985). The red cells indicate the existence of a significant $b$-value decrease. The number of red cells decreases as aftershocks are removed, but the decrease in $b$-value becomes more significant before the Miyagi event.

ing the $b$-value.

\section{Uniqueness of the Precursory Pattern \\ 5.1 Grid search}

By analyzing the seismic activity prior to the Miyagi event, we obtain an AIC as large as 7. Therefore, the significance level far exceeds $99 \%$. If the $b$-value is constant for most of area and period where no earthquake followed, we may be able to predict any forthcoming earthquake by searching similar patterns prospectively.

To test the uniqueness of the observed precursory pattern and study the feasibility of the earthquake prediction based on $b$-value change, we applied the obtained empirical model to various areas taken from throughout inland Japan. Our aim was to determine how often we would find a similar pattern in areas where no large earthquake had occurred in the preceding 1 year.

We mapped a grid covering all Japanese inland areas, with a $0.2^{\circ}$ spacing in both longitude and latitude. We then selected a node and gathered all shallow events within a circular area of $30 \mathrm{~km}$ in radius using the methodology of ZMAP (Wiemer, 2001). The events within the circle were analyzed to obtain the maximum likelihood estimate of parameters. We compared the two $b$-value models (2.4.1) 
and (2.4.2) using the AIC (Akaike, 1974) calculated for both models. Here we denote its difference between models as $\delta$ AIC. For convenience, we define SAIC as.

$$
\mathrm{SAIC}=\operatorname{sign}\left(B_{1}-B_{0}\right) \delta \mathrm{AIC} \operatorname{Boxcar}(\delta \mathrm{AIC} ; 0, \infty)
$$

The absolute value of SAIC is the difference in AIC between the two models, and the sign is positive when the $b$-value increases and negative when it decreases. If the null hypothesis has a lower AIC, the $b$-value change is not significant and is truncated to 0 .

\subsection{Results}

Figure 5 shows the map of changes in the $b$-value. The three plots depict the result with different declustering procedures. The map is colored according to the significance level of the existing of $b$-value decreases in the preceding 1 year.

Using the ETAS model, the significance level of the existence of $b$-value decreases near the Miyagi event was the highest among the 971 tests. Analysis of the catalog declustered by the Reasenberg's algorithm reveal that the level of significance is the third highest among all of the Japanese inland areas tested.

The minor differences between the results emerge as a result of the different approaches applied in the two declustering methods for removing the afterschocks. In most cases, the ETAS model removes more events as aftershocks than Reasenberg's algorithm. However, in areas where the aftershock sequence is represented well by the modified Omori's law (Utsu, 1961) and a special cluster coexists, the ETAS model does not remove special clusters (Tsukakoshi and Shimazaki, 2006).

We also conducted the same tests with three other models. Each model is created by shifting the time of Eq. (3) by 3 months.

$$
\begin{aligned}
B_{\text {background }}(t)= & B_{1} \operatorname{boxcar}(t ; 1997.75 .5,2001.75) \\
& +B_{2} \operatorname{boxcar}(t ; 2001.75,2002.75)
\end{aligned}
$$

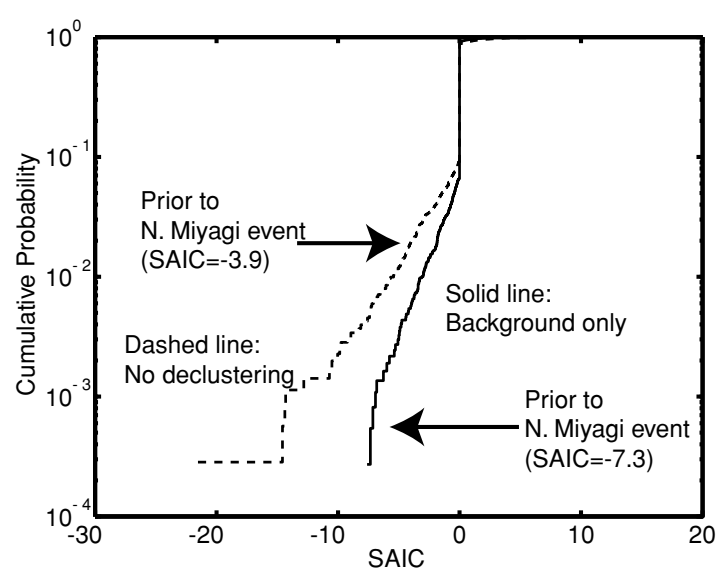

Fig. 6. Empirical probability density distribution of SAIC. The solid line shows the ascendant cumulative probability of SAIC obtained by studying $b$-value change of background seismicity. The dashed line shows that of all seismicity. The SAIC found prior to the Northern Miyagi event is indicated by arrows. The decrease in the background of the $b$-value is the second most significant among 3500 cells tested in this study.

$$
\begin{aligned}
B_{\text {background }}(t)= & B_{1} \operatorname{boxcar}(t ; 1998.0,2002.0) \\
& +B_{2} \operatorname{boxcar}(t ; 2002.0,2003.0) \\
B_{\text {background }}(t)= & B_{1} \operatorname{boxcar}(t ; 1998.25,2002.25) \\
& +B_{2} \operatorname{boxcar}(t ; 2002.25,2003.25) .
\end{aligned}
$$

We can not study older data because a large change occurred in the arrangement of the observatory stations in October 1997. Nonetheless, by considering the three models, we can increase the number of tests by a factor of 4 . The distribution of SAIC of more than 3500 tests is shown in Fig. 6. The significance prior to Miyagi event is the second highest in more than 3500 cells. The highest SAIC in this test is 7.6, which is a difference from the precursory SAIC of only 0.3 .

We also attempted a similar test without applying any declustering algorithm and obtained SAIC $=-3.9$ near the source region. The $b$-value change is statistically significant, but the significance is in 19th place out of the 971 cells. If we try another three time windows, the rank is the 70th place in the 3500 cells. There are more significant temporal $b$-value fluctuations if we include aftershocks.

\section{Discussion}

\subsection{The precursory period}

Several regression equations have been proposed for estimating the period of anomaly. Rikitake (1987) suggested

$$
\log _{10} T=0.44 M-0.55 \quad(T: \text { days }) .
$$

By substituting $M$ for 6.4 , we obtained approximately half a year. One case is slightly longer, but the scatter in his data is large.

A similar relation for the period of quiescence was given by Wyss and Habermann (1988).

$$
\log _{10} T=0.0+0.21 M \quad(T: \text { Month })
$$

or, changing the unit of $T$ to a year,

$$
\log _{10} T=0.21 M-1.08 \quad(T: \text { Year }),
$$

which is 1.75 years for $M=6.3$. The period of anomaly is close to our result of 1 year for the $b$-value and 2 years for seismicity level.

The precursory time of 1 year does not contradict with those studies. On the other hand, Enescu and Ito (2001) reported a longer period.

\subsection{Comparisons to precursory patterns of other earthquakes}

A similar pattern of $b$-value decrease is also found before the 2000 Western Tottori earthquake: there are precursory swarms on the source fault and the $b$-value is low (Shibutani et al., 2002). These findings also support the proposal that some earthquakes are preceded by an observable preparation process, such as pre-slip and fluid movement.

\subsection{On the level of the $b$-value}

Main et al. (1989) discussed the hypothesis that the critical failure in a large earthquake should occur at $b=0.5$. Our study shows a $b$-value of 0.7 . Considering that some unrelated events may be mixed in our sample, this study does not refuse the proposal of Main et al. (1989). However, the $b$-value is affected by the magnitude scale used, which is beyond the scope of our present investigation. 


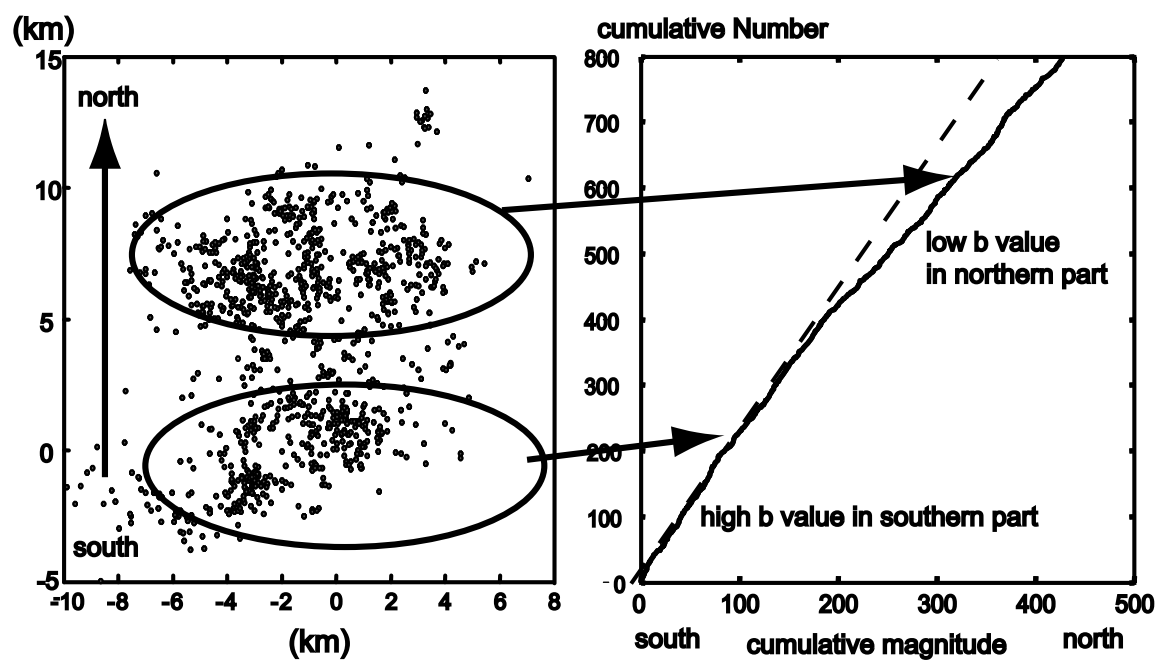

Fig. 7. The $b$-value change along the south-north section of the aftershock region. Based on the spatial distribution of the aftershocks, they can be divided into a northern cluster and southern cluster, respectively. The $b$-value of the southern cluster is higher than that of the northern part. On the other hand, the $b$-value inside the cluster seems to be rather homogeneous.

\subsection{One possible scenario on the Northern Miyagi se- quence}

The $b$-value of the aftershocks is lower at the northern part of the aftershock zone than at the southern part (Fig. 7), and the $b$-value at the northern cluster and southern cluster, respectively, is discrete. Based on the tri-axial compression test of Mogi (1962), a high $b$-value corresponds to a high heterogeneity of the medium. Thus, the crust may be highly heterogeneous in the south of the area. The low $b$-value character in the northern sector may be extended to the low $b$-value swarm area which resides to the north of the source region.

While there are significant differences in the $b$-value between clusters, the $b$-value inside the cluster seems to be homogeneous. The consistency of the $b$-value suggests the homogeneity of the crust of the area. In the scale of rupture size of the $M 2$ event, which is about several hundreds of meters, the area may be relatively homogenous inside each cluster.

\section{Conclusions}

The 2003 Northern Miyagi earthquake sequence was preceded by several precursory seismic activities. The $b$-value of seismicity near the source region decreased significantly during the year preceding the major event relative to the $b$-value of the preceding 5 years. When we remove aftershocks, the significance of the decrease of the $b$-value in this area seems to be equally relevant. This area has the highest significance among 971 other inland Japanese territories which were also tested in the same manner. When we conduct similar tests with four different time-windows, we find only one spot that exceeds the significance of the Miyagi event. We therefore conclude that monitoring the $b$ value change of background seismicity only may contribute to an estimation of the time-dependent seismic hazard.

By using the cumulative magnitude plot, we reveal that the $b$-value of the aftershocks seem to change spatially in a discontinuous manner, rather than in a gradual one. This may reflect several discontinuous heterogeneities of the crust. The spatial variety of the $b$-value suggests the existence of crustal patches larger than the source fault of M 2 class event.

Acknowledgments. We are grateful to Dr. Imoto at NIED and Prof. Ohtake, and Prof. Matuzawa at Tohoku University for their comments on the cumulative magnitude plot. We have used the earthquake catalog compiled by the Japan Meterological Agency. We have also used the GMT graphic package (Wessel and Smith, 1998) to plot some of the figures.

\section{Appendix A. Data Sources}

The earthquake catalog is provided by the Japan Meteorological Agency. This can be obtained from their FTP site. Contact johokan@redc-tk.eqvol.UkishUou.go.jp to obtain the data.

\section{References}

Akaike, H., A new look at the statistical model identification, IEEE Trans. Autom. Control, AC-19, 716-723, 1974.

Aki, K., Maximum likelihood estimates of $\mathrm{b}$ in the formula $\log \mathrm{N}=\mathrm{a}-\mathrm{bM}$ and its confidence limits, Bull. Earthq. Res. Inst., 43, 237-239, 1965.

Data Analysis section, Earthquake Prediction Information Division, Seismological and Volcanological Department, JMA, A study on the comparison of the new JMA magnitude with other magnitude, Q. J. Seismol., 67(1-4), 21-35, 2004.

Dempster, A. P., N. M. Laird, and D. B. Rubin, Maximum likelihood from incomplete data via the EM algorithm, J. R. Stat. Soc., B39, 1-39, 1977.

Enescu, B. and K. Ito, Some Premonitory Phenomena of the 1995 HyogoKen Nanbu (Kobe) Earthquake: seismicity, $b$-value and fractal dimension, Tectonophysics, 338, 297-314, 2001.

Faculty of Science, Tohoku University, Microearthquake Activity in and around the Tohoku District (May-October, 1990), Report of the coordinating committee for Earthquake Prediction, 45, 19-37, 1991.

Frohlich, C. and S. D. Davis, Teleseismic $b$ values: or, much ado about 1.0, J. Geophys. Res., 98, 631-644, 1993.

Gutenberg, B. and C. F. Richter, Frequency of Earthquakes in California, Bull. Seismol. Soc. Am., 34, 185-188, 1944.

Habermann, R. E. and M. S. Craig, Comparison of barkeley and calnet magnitude estimates as a means of evaluating temporal consistency of magnitudes in california, Bull. Seismol. Soc. Am., 78, 1255-1267, 1988.

Hainzl, S., G. Zoller, and J. Kurths, Earthquake clusters resulting from delayed rupture propagation in finite fault segments, J. Geophys. Res., 108(B1), 2013, doi:10.1029/2001JB000610, 2003.

Hikima, K. and K. Koketsu, Source processes of the foreshock, mainshock 
and largest aftershock in the 2003 Miyagi-ken Hokubu, Japan, earthquake sequence, Earth Planets Space, 56(2), 87-93, 2004.

Hirose, F., A. Nakamura, and A. Hasegawa, $b$-value Variation Associated with the rupture of Asperities-Spatial and Temporal Distributions of $b$-value East off NE Japan, Zishin II, 55, 249-260, 2002.

Imoto, M., Changes in the magnitude-frequency $b$-value prior to large $(M=6.0)$ earthquakes in Japan, Tectonophysics, 193, 311-325, 1991.

Imoto, M., A testable model of earthquake probability based on changes in mean event size, J. Geophys. Res., 108(B2), 10.1029/2002JB001774 07, 2003.

Inouye, W., On the seismicity in the epicentral region and its neighborhood before the Niigata earthquake, Q.J. Seismol., 29, 139-144, 1965.

JMA, Monthly Report on Earthquakes and Volcanoes in Japan, 48 pp., Jun 2003, 2003 (in Japanese).

Kagan, Y., Universality of the Seismic Moment-frequency Relation, Pure Appl. Geophys., 155, 537-573, 1999.

Kagan, Y. Y., Short-Term Properties of Earthquake Catalog and Models of Earthquake Source, Bull. Seismol. Soc. Am., 94(4), 1207-1228, 2004.

Kagan, Y. and L. Knopoff, Statistical search for non-random features of the seismicity of strong earthquakes, Phys. Earth Planet. Inter., 12, 291318, 1976.

Katsumata, A., Revision of the JMA Displacement Magnitude, Q. J. Seismol., 67, 1-10, 2004

Kelis-Borok, V. I., L. Knopoff, I. M. Rotvain, and T. M. Sidorenko, Bursts of Seismicity as Long-Term Precursors of Strong Earthquakes, J. Geophys. Res., 85(B2), 803-811, 1980.

Kellis-Borok, V. I. and V. G. Kossobokov, Time of Increased Probability of strong Earthquakes $(M>=7.5)$ Diagnosed $b$ Algorithm M8 in Japan and Adjacent Territories, J. Geophys. Res., 95(B8), 12413-12422, 1990.

Knopoff, L., Y. Y. Kagan, and R. Knopoff, $b$ values for foreshocks and aftershocks in real and simulated earthquake sequences, Bull. Seismol. Soc. Am., 72, 1663-1676, 1982.

Lei, X.-L., K. Masuda, O. Nishizawa, L. Jouniaux, L. Liu, W. Ma, T. Satoh, and K. Kusunose, Detailed analysis of acoustic emission activity during catastrophic fracture of faults in rocks, J. Struct. Geol., 26, 247-258, 2004.

Li, Q., J. Cen, L, Yu, and B. Hao, Time and space scanning of the $b$-valuea method for monitoring the development of catastrophic earthquakes, Acta Geophys. Sinica, 21, 101-125, 1978.

Lombardi, A. M., The Maximum Likelihood Estimator of $b$-value for Mainshocks, Bull. Seismol. Soc. Am., 93(5), 2082-2088, 2003.

Main, I., Statistical physics, seismogenesis, and seismic hazard, Rev. Geophys., 34(4), 433-462, 1996.

Main, I. G., P. G. Meredith, and C. Jones, reinterpretation of the precursory seismic $b$-value anomaly from fracture mechanics, Geophys. J. Int., 96 , 131-138, 1989.

Mashiko, N. and S. Noguchi, Comparison of hypocenter data between the NIED Kanto-Tokai network, Hi-net and JMA catalog (part2), Programme and Abstracts, the Seismol. Soc. Japan, 2003, fall meeting, A049, 2003 (in Japanese).

Mogi, K., Study of Elastic Shocks caused by the Fracture of Heterogeneous Material and its Relation to Earthquake Phenomena, Bull. Earthq. Res. Inst., 40, 125-173, 1962.

Mogi, K., Some features of recent seismic activity in and around Japan, 2, Activity before and after great earthquakes, Bull. Earthq. Res. Inst., 47, 395-417, 1969.

Mogi, K., Downward Migration of Seismic Activity Prior to Some Great Shallow Earthquakes in Japanese Subduction Zones-A Possible Intermediate-term Precursor, Pure Appl. Geophys., 126, 447-464, 1988

Ogata, Y. and K. Katsura, Space-time Point-process models for earthquake occurrences, Ann. Inst. Statist. Math., 50(2), 379-402, 1989.

Ohnaka, M. and K. Mogi, Frequency Characteristics of Acoustic Emission in Rocks Under Uniaxial Compression and Its Relation to the Fracturing Process to Failure, J. Geophys. Res., 87(B5), 3873-3884, 1982.

Ohta, K., Y. Fujiwara, and K. Maeda, Spatiotemporal variation of minimum magnitude of completeness in the JMA Catalog, Q. J. Seismol., 65(1-4), 111-122, 2004

Ohtake, M., Earthquake prediction based on the seismic gap with special reference to the 1978, Oaxaca, Mexico earthquake, Rept. Natl. Cent.
Disaster Prev., 23, 65-110, 1980.

Reasenberg, P., Second-order moment of California seismicity, 19691982, J. Geophys. Res., 78, 5479-5495, 1985.

Rikitake, T., Earthquake precursors in Japan: precursor time and detectability, Tectonophysics, 136, 265-282, 1987.

Rundle, J. B., W. Klein, D. L. Turcotte, and B. D. Malamud, Precursory seismic activation and critical-point phenomena, Pure Appl. Geophys., 157, 2165-2182, 2000.

Sammonds, P. R., P. G. Meredith, and I. G. Main, Role of pore fluids in the generation of seismic precursors to shear fracture, Nature, 359, 228230, 1992.

Scholz, C. H., The frequency-magnitude relation of micro fracturing in rock and its relation to earthquakes, Bull. Seismol. Soc. Am., 58, 388415, 1968.

Sekiya, H., The Seismicity Preceding Earthquakes and its Significance to Earthquake Prediction, Zishin II, 29, 299-311, 1976.

Shibutani, T., S. Nakao, R. Nishida, F. Takeuchi, K. Watanabe, and Y. Umeda, Swarm-like seismic activity in 1989, 1990 and 1997 preceding the 2000 Tottori-ken Seibu Earthquake, Earth Planets Space, 54, 831845, 2002.

Smith, W. D., The $b$-value as an earthquake precursor, Nature, 289, 136139, 1981.

Smith, W. D., Evidence for precursory changes in the frequency-magnitude $b$-value, Geophys. J. R. Astr. Soc., 86, 815-838, 1986.

Smith, W. D., Resolution and significance assessment of precursory changes in mean earthquake magnitudes, Geophys. J. Int., 135, 515$522,1999$.

Sykes, L. R. and S. C. Jaume, Seismic Activity on Neighbouring Faults as a long-term Precursor to Large Earthquakes in the San Francisco Bay Area, Nature, 348, 595-599, 1990.

Tsukakoshi, Y. and K. Shimazaki, Temporal behavior of the background seismicity rate in central Japan 1998 to mid-2003, Tectonophysics, 417, 155-168, 2006.

Tsukakoshi, Y., S. Naito, and N. Ishida, Probablistic characteristics of stress changes during cereal snack puncture, J. Texture Stud., 38, 220 235, 2007.

Tsukakoshi, Y., S. Naito, and N. Ishida, Fracture Intermittency during a puncture test of cereal snacks and its relation to porous structure, Food Res. Int., 2008 (in press).

Utsu, T., A Statistical Study on the Occurrence of Aftershocks, Geophys Mag., 30, 521-605, 1961.

Utsu, T., A Method for Determining the Value of $b$ in a Formula log $\mathrm{n}=\mathrm{a}-\mathrm{bM}$ Showing the Mangitude-frequency Relation for Earthquakes, Geophys. Bull. Hokkaido Univ., 13, 99-103, 1965 (in Japanese).

Utsu, T., Introductions to Seismicity, Surijishingaku (Mathematical Seismology) (VII), Inst. Stat. Math., 139-157, 1992 (in Japanese).

Varnes, D. J., Predicting Earthquakes by Analyzing Accelerating Precursory Seismic Activity, Pure Appl. Geophys., 130, 661-686, 1989.

Wessel, P. and W. H. F. Smith, New improved version of generic mapping tools released, Eos Trans. AGU, 79(47), 579, 1998.

Wiemer, S., A Software package to analyze seismicity: ZMAP, Seismol. Res. Lett., 72(2), 373-382, 2001.

Wyss, M. and W. H. K. Lee, Proc. Conf. In Tectonic Problems of the San Andreas Fault System, 13, 24-42, Stanford University, 1973.

Wyss, M. and R. E. Habbermann, Precursory seismic quiescence, Pure Appl. Geophys., 126, 319-332, 1988.

Yagi, Y., Y. Ito, and S. Sekine, Fore-shock, Mainshock, and the largest after-shock associated with the July 262003 Miyagi-ken-hokubu, Japan, earthquake, Programme and Abstracts, the seismological society of Japan 2003, fall meeting, A086, 2003.

Yamashita, T. and L. Knopoff, Model for intermediate-term precursory clustering of earthquakes, J. Geophys. Res., 97, 19873-19879, 1992.

Zhuang, J., D. Vere-Jones, and Y. Ogata, Analyzing earthquake clustering features by using stochastic reconstruction, J. Geophys. Res., 109(B5), doi:10.1029/2003JB002879, 2004

Y. Tsukakoshi (e-mail: yoshiki.tsukakoshi@gmail.com) and K. Shimazaki 